\title{
Hypertension and facial palsy in middle aortic syndrome
}

\author{
V E Lewis, D S Peat, E J Tizard
}

\begin{abstract}
A female infant presented with facial palsy and was found to be severely hypertensive. Plasma renin activity was raised and an angiogram showed middle aortic syndrome. This condition is of unknown aetiology, but positive antineutrophil cytoplasmic antibodies may indicate a vasculitis which heals by intimal fibrosis, causing the observed findings.

(Arch Dis Child 2001;85:240-241)
\end{abstract}

Keywords: hypertension; facial palsy; middle aortic syndrome

\section{Case report}

An 11 month old girl presented with a three week history of irritability, lethargy, and a right sided lower motor neurone facial palsy. She had a faint petechial rash over her legs which had prompted her general practitioner to diagnose Henoch-Schönlein purpura. Past medical, perinatal, and family histories were unremarkable. On examination a four limb blood pressure of $210 / 160 \mathrm{~mm} \mathrm{Hg}$ was recorded. The femoral pulses were normal at presentation and have remained so. Fundoscopy revealed bilateral retinal haemorrhages and tortuous vessels. There was no arthropathy.

Initial investigations showed a normal renal profile and full blood count. There was moderate haemolysed blood and protein on urinalysis with a sterile urine culture. Urinary catecholamines were within normal limits. Serum renin and aldosterone assays were requested. Viral serology, complement, and chromosomes were all normal. An autoimmune profile showed weakly positive antinuclear and antismooth muscle antibodies (titres $1 / 80$ and $1 / 160$ respectively), a positive IgG antineutrophil cytoplasmic antibody (ANCA) with an atypical "flat" staining pattern (titre 1/320), and a positive IgA ANCA. It was not possible to identify the antigen. Renal ultrasound showed a large (bipolar length $80 \mathrm{~mm}$ ), bright right kidney and a normal (bipolar length 67 $\mathrm{mm}$ ) left kidney. No suprarenal masses were evident and Doppler studies suggested good bilateral flow in the renal arteries. There was no dilatation of the urinary tract. Echocardiography showed left ventricular and septal hypertrophy with good ventricular function and a normal aortic arch.

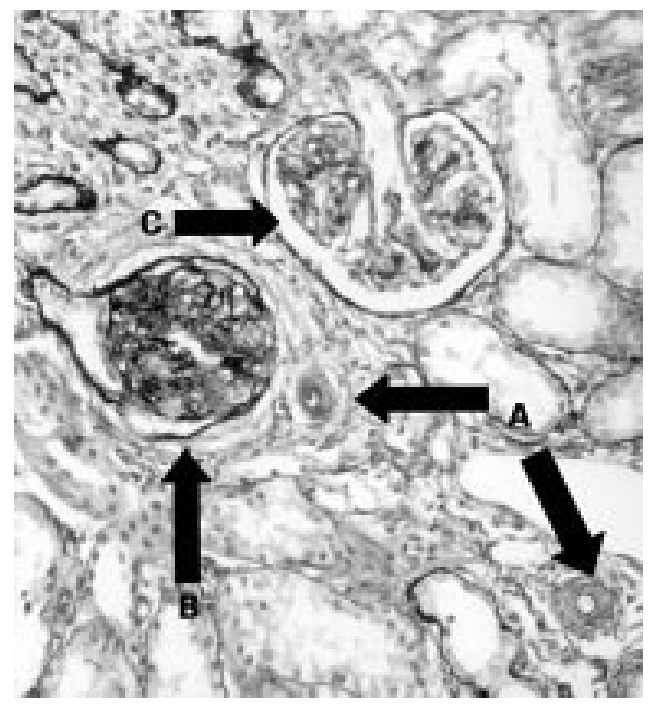

Figure 1 Renal biopsy showed hypertensive changes in arterioles (arrows $A$ ) with sclerosed glomerulus (arrow B). The viable tuft (arrow $C$ ) is within normal limits (original magnification $\times 200$, methanamine silver stain).

Percutaneous biopsy of the right kidney (fig 1) showed sclerosis in five of 43 glomeruli. The arterioles showed hyaline sclerosis in keeping with hypertension. Immunofluorescence showed deposition of C3 and granular $\operatorname{IgM}$ in the glomerular basement membrane with no IgA present. A technetium 99mdimercaptosuccinic acid (DMSA) scan showed decreased uptake in the upper and mid portions of the left kidney with normal right sided appearances. Differential function was abnormal with only $25 \%$ on the left side.

The blood pressure was gradually brought under control with intravenous labetolol. Management with oral medication required a combination of nifedipine, angiotensin converting enzyme inhibitors, and $\beta$ blockers.

Plasma renin activity was reported to be $57 \mathrm{pmol} / \mathrm{ml} / \mathrm{h}$ with an aldosterone concentration of $2330 \mathrm{pmol} / 1$ (normal plasma renin activity and aldosterone at St Mary's Hospital, Paddington $<10 \mathrm{pmol} / \mathrm{ml} / \mathrm{h}$ and $<2000 \mathrm{pmol} / 1$ respectively). These results suggested a renovascular cause of hypertension and an angiogram was performed.

The angiogram (fig 2) showed a mid aortic narrowing, maximal just distal to the origin of the right renal artery. Collateral branches were visualised, arising from the inferior adrenal 


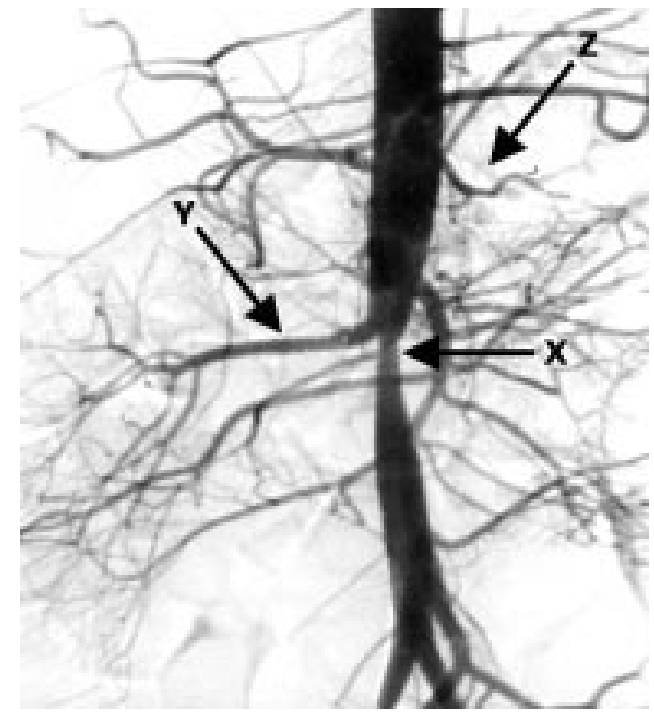

Figure 2 Angiogram showing mid aortic stenosis (arrow $X$ ). Right renal artery (arrow Y) is smaller than expected with no left renal artery shown. Artery arising directly from aorta (arrow Z) supplies left upper pole only.

artery. On the left side a small artery to the left upper pole was identified, arising directly from the aorta. Notable collateral branches were seen from the vertebral arteries. No vessels were shown supplying the lower pole of the left kidney. These changes are diagnostic of the middle aortic syndrome. The absence of a differential blood pressure between upper and lower limbs has never been satisfactorily explained. After the angiogram the patient was carefully re-examined and was still felt to have normal femoral pulses. She has never had symptoms or signs of under perfusion of the lower limbs.

The extent of the vascular abnormalities excluded surgical treatment options and with normal renal function, a conservative management plan was adopted. Although difficult to control in the early phase of the illness, systolic and diastolic blood pressures have remained within the 95th centile for age on a combination of $\beta$ blockers and angiotensin converting enzyme inhibitors. The facial palsy resolved completely within six months and, at the age of 6 years, she is on the 90th centile for height and weight and developmentally advanced. She remains asymptomatic with normal renal function.

\section{Discussion}

Facial palsy in children has numerous aetiologies and always warrants full investigation. The association with hypertension was first described by Moxon in 1869. ${ }^{1}$ Subsequent series $^{23}$ have suggested that hypertension is responsible for $3-17 \%$ of lower motor neurone facial palsies. Furthermore, this specific neurological sign may be the only presenting abnormality in a hypertensive child. The mechanism of nerve damage is thought to be haemorrhage into the facial canal; it is usually unilateral and, with the return to normotension, resolves completely within weeks to months.

Middle aortic syndrome is a rare condition of unknown aetiology, characterised by tubular stenosis of the abdominal aorta and its visceral branches; it was first described by Sen et al in 1969 in a series of young females. ${ }^{4}$ There are similarities with Takayasu's arteritis; some authors regard them as being synonymous. ${ }^{5}$

In 1976 Kaufman et al presented a series of cases of renovascular hypertension, some of which fitted the description of middle aortic syndrome. ${ }^{6}$ They hypothesised that arteritis occurred during an acute flu like illness and that healing in the form of fibrosis occurred after a variable latent period. This was based on histology of resected arteries at the time of surgical revascularisation, but immunocytochemistry failed to show the presence of active inflammation.

In our reported case we believe that despite the lack of a demonstrable antigen, the high ANCA titre suggests that there may have been an underlying vasculitis. This appears to be the first case in which this has been shown.

In conclusion, lower motor neurone facial palsy in children may be secondary to hypertension which warrants exhaustive investigation. Middle aortic syndrome is a rare cause of renovascular hypertension and a definitive diagnosis can only be made angiographically. The aetiology is obscure, but may involve an autoimmune large vessel vasculitis which heals by intimal fibrosis, resulting in the described arterial narrowings.

We thank Professor Dillon at Great Ormond Street Hospital for the angiograms.

1 Moxon M. Apoplexy into canal of fallopius in a case of Bright's disease, causing facial paralysis. Trans Pathol Soc London 1869;20:420-2.

2 Siegler RL, Brewer ED, Corneli HM. Hypertension first seen as facial paralysis: case reports and review of the literature. Paediatrics 1991;87:387-9.

3 Lloyd AVC, Jewitt DE, Lloyd Still JD. Facial paralysis in children with hypertension. Arch Dis Child 1966;41:292-4.

4 Sen PK, Kinare SG, Engineer SD, et al. The middle aortic syndrome. Br Heart f 1963;25:610-18.

5 Pagni S, Denatale RW, Boltax RS. Takayasu's arteritis: the middle aortic syndrome. Am Surg 1996;62:409-12.

6 Kaufman JJ, Ehrlich RM, Dornfeld L. Immunologic considerations in renovascular hypertension. $\mathcal{F}$ Urol 1976;116: 142-7. 